\title{
The future is now: carrier screening for all populations
}

\author{
Beth A. Pletcher, MD, ${ }^{1}$ Susan J. Gross, $M D,{ }^{2}$ Kristin G. Monaghan, PhD, ${ }^{3}$ Deborah A. Driscoll, MD, ${ }^{4}$ \\ and Michael S. Watson, $P h D^{5}$
}

Before the introduction of panethnic cystic fibrosis gene carrier screening, population-based genetic carrier screening to inform reproductive decision-making was based on the at-risk individual's ethnic and geographic background. In the Ashkenazi Jewish population, carrier screening programs continue to expand as more genes and mutations are identified in diseases for which this population is at increased risk. However, as more diseases are added to these panels, some carrier states in the targeted populations begin to approach the prevalence in the general population leading one to consider whether it is more appropriate to employ panethnic screening panels for these conditions rather than subpopulation-targeted panels. Further, the burden of the diseases being considered for carrier screening is broadening from what initially was a small group of neurodegenerative diseases that were lethal in early childhood to conditions with arguably less burden and more variability. Questions about the clinical utility of using ethnicity or other surrogates of ancestral geographic origin to direct clinical services are also increasing. As population admixture continues to grow in the United States, the utility of defining individuals on this basis can only decrease.

As technology continues toward very high throughput, low cost, and multiplexed applications, we are rapidly approaching a scenario similar to that for newborn screening several years ago. Questions abound as to which conditions should be considered for screening and the criteria by which this is done; who should be offered different screening panels and how are those groups are defined? However, many of those decisions will have to be balanced against the inherent trade-offs in clinical performance of tests for an ever increasing number of potential analytical targets.

To gain a better understanding of the issues involved in developing carrier screening programs in the United States and to open a dialog with communities that have typically had much influence over decisions as to what sorts of voluntary screening

\footnotetext{
From the ${ }^{1}$ Department of Pediatrics, University of Medicine and Dentistry of New Jersey New Jersey Medical School, Newark, New Jersey; ${ }^{2}$ Division of Reproductive Genetics, Department of Obstetrics and Gynecology and Women's Health, Albert Einstein College of Medicine, Bronx, New York; ${ }^{3}$ Department of Medical Genetics, Henry Ford Hospital, Detroit, Michigan; ${ }^{4}$ Department of Obstetrics and Gynecology, University of Pennsylvania Hospital, Philadelphia, Pennsylvania; ${ }^{5}$ American College of Medical Genetics, Bethesda, Maryland.

Michael S. Watson, ACMG, 9650 Rockville Pike, Betlesda, MD 20904. E-mail: mwatson@ acmg.net.

Disclosure: The authors declare no conflict of interest.

Submitted for publication July 3, 2007.

Accepted for publication September 10, 2007.
}

DOI: 10.1097/GIM.0b013e31815f5934 programs would meet their needs, a meeting of stakeholders and genetics professionals was developed.

On November 19, 2006, the conference titled "Genetic Carrier Screening: Moving Population Genetics from Theory to Practice" was held in the Bronx, New York. It was cosponsored by the American College of Medical Genetics and Jacobi Hospital. This meeting was unique in that a wide array of participants came together to discuss a host of current, and potential future screening targets. This meeting, as well as future meetings of this kind, may be used to determine the most appropriate diseases to include in general population and race or ethnic-specific genetic testing. There were presentations on specific disorders given by scientists and physicians, as well as population perspectives regarding carrier screening. What ensued was a dialogue among physicians, scientists, patients, parents, advocates, and diverse community members who are all stakeholders in this very promising, yet uncertain endeavor. Here we will highlight some of the issues that came from these presentations and discussions. Of note, the choice of specific community groups who addressed this meeting was not meant to be comprehensive in any way. Rather, they represent a cross section of various population groups who have garnered extensive experience with genetic issues over the past several years. Certainly there were common themes that would seem to apply to community outreach regardless of ethnic or racial background. However, there remain other communities whose concerns were not addressed specifically in this meeting and who will need to be heard from in the future.

\section{NEWBORN AND CARRIER SCREENING (M. WATSON)}

Although the conference was dedicated to carrier screening, the meeting opened with an overview of the American newborn screening experience. We have much more experience with newborn screening (NBS) compared with general population carrier screening. Although NBS remains a moving target with rapid expansion of tests mandated by individual states for all newborns, there are certainly particular facets of the NBS program where we can integrate what we have learned into general population carrier screening.

For decades, general population carrier screening was based on clinical validity and clinical utility to direct services based on ethnicity, race or other social factors that may lead to particular conditions being more common in a particular group. The two major examples have been carrier screening for infantile lethal neurodegenerative disorders in the Ashkenazi Jewish community and hemoglobinopathies in the African American population. However in the United States, ethnicity and race 
are difficult to ascertain due to admixture. Therefore, we will likely need to move toward screening more broadly in our multicultural and multiethnic society. Furthermore, with advances in genetic knowledge and technologies, carrier screening for additional disorders such as cystic fibrosis have now become part of primary care.

Although carrier testing and NBS differ in many respects, broad-based panethnic testing and efficient use of current technologies are two areas where our experience with NBS can be of considerable help. Most importantly, there needs to be some standardization of criteria to select population-based screening tests, similar to the ones used for NBS. To select appropriate tests, we need to understand the burden and natural history of each condition, the inheritance and carrier frequency, and genotype-phenotype correlations. Further, with a nearly unlimited number of gene mutations that might be considered for carrier screening panels, fundamental questions about the performance of tests (e.g., true positives and false positives) and how one's follow-up results must be considered.

\section{COMMUNITY SUPPORT (C. SCRIVER)}

To develop a successful screening program, one always needs to consider a community perspective. Ideally a screening program should be initiated by the community being served, so that it will be well accepted by the targeted population. If a program strives for community "buy in," then the program might not be perceived as something that is foisted on the participants, and is likely to meet with less resistance and ensure participation. Although in the United States we have generally avoided genetic screening programs targeting high school students, this approach has been extremely successful in Canada. Dr. Scriver presented basic principles related to successful programs which hinge on mutual understanding and partnership with the community.

\section{CYSTIC FIBROSIS (D. DRISCOLL)}

Population-based carrier screening for cystic fibrosis (CF) arrived with the release of the ACOG/ACMG Clinical and Laboratory Guidelines in 2001 and may serve as a model for future panethnic population preconception carrier screening endeavors in the United States. Critical to its introduction was recognition that nongenetics-trained providers are on the frontline of the delivery of this service, thereby necessitating the standardization of testing protocols and the development of educational materials for providers. CF carrier screening has been well integrated into standard prenatal care in the United States. However, there remain some challenges and opportunities, with a suggestion that physicians, other health care providers, and consumers still need additional education about screening, including the meaning of a negative or positive test result. Physicians need simple and straightforward guidelines to follow in order to fully incorporate CF screening into their practices. Furthermore, we need to ensure that all laboratory re- ports are simple and understandable in order to prevent confusion and misinterpretation.

\section{SPINAL MUSCULAR ATROPHY (D. HEINE)}

Population carrier screening for spinal muscular atrophy (SMA) may be an appropriate disease to consider for population-based preconception carrier screening. SMA affects individuals of all ethnic and racial backgrounds equally. The carrier frequency is estimated to be about 1 in 40 , with two affected infants born every day in the United States. SMA is a severe and often fatal disease where the vast majority of affected individuals are homozygous for the loss of exon 7 in the SMN1 gene. Severity of the disorder may be predicted based on the number of copies in the highly related SMN2 gene, thus potentially allowing for phenotype-genotype correlation. Preconception and prenatal screening are now technically feasible because a molecular test is available to detect the common mutation that accounts for approximately $95 \%$ of the SMA alleles. Before moving forward with this testing, there is a need for pilot data looking at a number of issues: including accurate carrier frequency, genotype-phenotype correlation, the ability to perform testing on large numbers of prospective parents with automation of the assay, and the best way to educate providers and patients about this screening test. There is considerable interest nationally in pilot efforts for SMA carrier screening. In light of the success of CF carrier screening that is being offered to a broad range of patients, a similar model could be adapted for SMA carrier screening in the future.

\section{CARRIER SCREENING WITHIN THE ASHKENAZI JEWISH POPULATION (H. OSTRER, S. MICHELMAN, R. YUDENFREUND GLASER)}

Because of endogamy and genetic drift, there is a higher carrier rate for certain disorders and a limited number of mutations accounting for the majority of deleterious alleles in this population. Carrier screening has been used to identify couples at risk for having affected children. As a result, there have been significant reductions in the births of infants with these conditions among Ashkenazi Jews.

Survey data have suggested that for the Jewish community, the cost of testing is often less important than the severity or impact of the condition. Many Ashkenazi Jewish patients prefer a very broad menu of tests; prospective parents often state that statistics mean very little to them and the incidence of any individual condition is not a major factor in their decision to have or not have carrier screening if it is available. Mr. Stanley Michelman and Mrs. Randi Yudenfreund Glaser representing the Jewish Genetic Disease Consortium, an umbrella organization of the various Jewish genetic disease community support groups, shared a message on behalf of the consortium as well as their own very personal perspectives on carrier screening in the Jewish community. The goals of the Consortium are to promote education and screening for a more comprehensive panel of disorders. For Ashkenazi Jewish individuals, as 
well as other consumers, we need to ensure that the patients are fully educated about testing, so that informed consent is meaningful. Likewise, cultural issues need to be taken into account such that testing strategies are tailored to the marriage patterns and religious practices of the individuals being offered screening. Conference participants suggested that any new clinical guidelines should address screening of couples of mixed ethnicity and assuring adequate informed consent in the context of multiplex testing.

In this issue of Genetics in Medicine there are two guidelines regarding screening for couples and individuals of Ashkenazi Jewish ancestry. "Technical Standards and Guidelines for Reproductive Screening in the Ashkenazi Jewish Population" 1 (page 57) was prepared by the Laboratory Quality Assurance Committee. This is an up-to-date resource developed to provide guidance to clinical laboratories and laboratory directors who may be performing these tests. The parallel clinical guideline "Carrier Screening in Individuals of Ashkenazi Jewish Descent" (page 54), prepared by the Professional Practice and Guidelines Committee, serves to put these laboratory issues into a clinical context. ${ }^{2}$

Whereas population-based carrier testing in the Ashkenazi Jewish population has been the focus of a great deal of energy and attention in recent years, there are other Jewish populations and other endogamous non-Jewish populations at risk for other serious genetic conditions. These populations might be candidates for ethnic-specific, as well as panethnic testing panels.

\section{THE AFRICAN AMERICAN PERSPECTIVE (R. NAGEL, V. DUBERRY ADEMU-JOHN)}

As outlined by Ms. Verna DuBerry Ademu-John, Program Coordinator at the Brookdale Comprehensive Pediatric Sickle Cell Program, carrier screening in the African American community brings up a number of additional issues. The "legacy of distrust" is a part of the outlook on sickle cell screening in this country and, within this community, remains an important concern. It would behoove individuals and groups in the position to make an impact on carrier screening to take this into consideration and approach screening in a holistic and sensitive manner. Another major point is that the term "African American " does not cover many of the groups affected by sickle cell disease and trait. Many do not consider themselves African American, for example, individuals who have recently arrived from the continent of Africa and from the Caribbean. Ms. DuBerry Ademu-John suggests that a better term would be "people from the African Diaspora in America." The presentation did stress that, overall, this community is very interested in genetic education and the benefits of current medical genetic services. However, there was considerable concern as to how education about screening was being provided in the medical setting, if at all. Furthermore, there were legitimate issues raised regarding overall lack of genetic knowledge within the primary care community itself. The identification of sickle cell carrier status during a pregnancy often causes distress be- cause parents may be unaware of a risk before conception or may not be aware that they have even undergone screening. Parents and prospective parents in the African American community can and wish to make their own decisions about screening once they are armed with knowledge. It is also not necessary to wait for a pregnancy to initiate screening. Children born outside of the United States could have testing done before entering kindergarten, high school, or college. From this community's perspective, models for earlier preconception or childhood screening should be undertaken and funded through community-based programs with the understanding that the positive impact can be maximized through grassroots and community-based initiatives. Other suggestions to improve care include providing NBS test results to all parents of children who screen positive for hemoglobinopathy carrier status, and ensuring that sickle cell test results become part of all students' health records.

\section{THE LATINO PERSPECTIVE (A. IGLESIAS, A. NICASIO)}

As of July 2005, 42.7 million Latinos resided in the United States, which represents approximately $14 \%$ of the population. By 2050 , the projected Latino population will be 102.6 million (24\%) or almost a quarter of the US population. Latinos may be of any race, and derive from multiple ethnic backgrounds, which will certainly have a significant impact on the design of any expanded genetic screening panels. For Latinos, health issues are often influenced by variable combinations of educational backgrounds, religious traditions, folk beliefs, family traditions, and trust in Western medicine and science. Latinos' particular characteristics suggest that genetic screening policies and attendant issues related to understanding and participation should be tailored to their needs. The mandatory nature of newborn screening often puts Latinos in an unwanted position, suggesting that customized counseling is very important. Education, in a broad sense, will probably be the cornerstone for success for future policy design and implementation. Making strides in areas such as education, income, health insurance, and acculturation will influence Latinos' screening practices. Educational interventions for health care providers in the prenatal and postnatal settings who care for Latinos will also be helpful. Increasing the numbers of health care providers of Latino origin, as well as counselors familiar with "culturally sensitive" issues is not only desirable, but necessary.

\section{LEGAL CONSIDERATIONS (N. ELSTER)}

In this day and age, one always needs to be cognizant of legal issues in testing and care of patients. With genetic screening in particular, several important legal issues arise such as insurance and employment discrimination, privacy, confidentiality, and informed consent- - both for testing and treatment. Many states have laws in place to prevent insurance or employment discrimination based on genetic test results; three states have laws specific to carrier screening. Seventeen states have laws that penalize providers for violating a patient's privacy regard- 
ing genetic information and 27 states require consent for disclosure of genetic information to a third party. Whereas case law has set a precedent for antidiscrimination in relationship to genetic testing, there are still some areas where things are not as clear-cut, such as duty to disclose. There are two proposed pieces of legislation that deserve special attention: S.976 Genomics and Personalized Medicine Act of 2007, HR.493 and S.358 Genetic Information Nondiscrimination Act (GINA) banning discrimination against US residents based on genetic test results which will be important to follow this next year.

\section{NEXT STEPS}

Following this meeting, we reflected upon what we had learned from the presentations and how best to use this information in the expansion of population carrier screening. We need to identify the communities we hope to serve and take into account the needs of the families in these various communities. For populations where genetics is not a high priority (i.e., the Latino population) we need to find the best way to educate individuals about their options so they can make informed decisions. We need to determine who in a busy medical practice has the time and skills necessary to counsel patients where time and even requisite skills may be limited. Communities are finding nonphysicians to supplement more classical models of health care. As the technology moves forward there are innumerable conditions, including SMA and Fragile X, which may be amenable to multiplex platforms. We need to answer questions such as "How common should a condition be in order to include it as a carrier screening test?" or "Is the prevalence of a disorder only one factor that needs to be balanced against clinical impact and cost of testing?". Variable carrier rates in broad populations make race/ethnic-based recommendations even more difficult to develop (i.e., the carrier rate for sickle cell in Hispanic populations varies greatly de- pending on country of origin) such that panethnic testing may become a more viable option in the forever changing American landscape.

When considering population-based carrier screening in a general way, it is essential to be very thoughtful as guidelines are developed and to seek the input of both professionals and community members. Deciding which conditions should be added, and when, is difficult at best. This meeting is just the beginning of our journey to establish a national agenda to raise awareness about, and develop guidelines for, US carrier screening. We hope that this meeting is the first of a number of collaborative activities that will allow us to provide the best care for all of our patients, regardless of ethnicity, race, socioeconomic status, or insurance coverage. We realize that the future of population-based carrier screening is now, and the train has already left the station. We all need to hop on board in order to arrive safely at our final destination.

\section{ACKNOWLEDGMENTS}

Listed below are the invited speakers who gave of their time and shared their perspectives and expertise during the "Genetic Carrier Screening: Moving Population Genetics from Theory to Practice" conference held on November 20, 2006: Verna DuBerry Ademu-John, MS, Deborah A. Driscoll, MD, Nanette Elster, JD, MPH, Randy Yudenfreund Glaser, Susan J. Gross, MD (Co-Chair), Deborah Heine, Alejandro D. Iglesias, $\mathrm{MD}$, Stanley Michelman, Ronald Nagel, MD, Andel Nicasio, MSEd, Harry Ostrer, MD, Charles R. Scriver, MD, Allen M. Spiegel, MD, Michael S. Watson, $\mathrm{PhD}$ (Co-Chair).

\section{References}

1. Monaghan KG, Feldman GL, Palomaki GE, Spector EB, et al. Technical standards and guidelines for reproductive screening in the Ashkenazi Jewish population. Genet Med 2008;10:57-72.

2. Gross SJ, Pletcher BA, Monaghan KG. Carrier screening in individuals of Ashkenazi Jewish descent. Genet Med 2008;10:54-56. 